\title{
Psychoactive pharmaceuticals at environmental concentrations induce in vitro gene expression associated with neurological disorders
}

\author{
Gaurav Kaushik ${ }^{1,2,3^{*}}$, Yu Xia ${ }^{4}$, Luobin Yang ${ }^{1}$ and Michael A. Thomas ${ }^{1}$
}

From 12th Annual Biotechnology and Bioinformatics Symposium (BIOT-2015)

Provo, UT, USA. 10-11 December 2015

\begin{abstract}
Background: A number of researchers have speculated that neurological disorders are mostly due to the interaction of common susceptibility genes with environmental, epigenetic and stochastic factors. Genetic factors such as mutations, insertions, deletions and copy number variations (CNVs) are responsible for only a small subset of cases, suggesting unknown environmental contaminants play a role in triggering neurological disorders like idiopathic autism. Psychoactive pharmaceuticals have been considered as potential environmental contaminants as they are detected in the drinking water at very low concentrations. Preliminary studies in our laboratory identified gene sets associated with neuronal systems and human neurological disorders that were significantly enriched after treating fish brains with psychoactive pharmaceuticals at environmental concentrations. These gene expression inductions were associated with changes in fish behavior. Here, we tested the hypothesis that similar treatments would alter in vitro gene expression associated with neurological disorders (including autism) in human neuronal cells. We differentiated and treated human SK-N-SH neuroblastoma cells with a mixture (fluoxetine, carbamazepine and venlafaxine) and valproate (used as a positive control to induce autism-associated profiles), followed by transcriptome analysis with RNA-Seq approach.

Results: We found that psychoactive pharmaceuticals and valproate significantly altered neuronal gene sets associated with human neurological disorders (including autism-associated sets). Moreover, we observed that altered expression profiles in human cells were similar to gene expression profiles previously identified in fish brains.

Conclusions: Psychoactive pharmaceuticals at environmental concentrations altered in vitro gene expression profiles of neuronal growth, development and regulation. These expression patterns were associated with potential neurological disorders including autism, suggested psychoactive pharmaceuticals at environmental concentrations might mimic, aggravate, or induce neurological disorders.
\end{abstract}

\footnotetext{
*Correspondence: gkaushik@ucdavis.edu

'Department of Biological Sciences, Idaho State University, Stop 8007, 9215

8th Ave, Pocatello, ID 83209-8007, USA

2Department of Medical Pathology and Laboratory Medicine, University of

California at Davis, Davis, CA 95817, USA

Full list of author information is available at the end of the article
} 


\section{Background}

There are approximately 3000 synthetic chemicals known to interact with humans through air, water, and food $[1,2]$. These chemicals might serve as environmental contaminants and trigger neurological disorders, such as autism spectrum disorders (ASD), in genetically susceptible individuals [3-5]. Among this diverse group of environmental contaminants, we focused on pharmaceuticals and personal care products (PPCPs) due to their tendency to contaminate environmental water systems [2]. PPCPs include psychoactive pharmaceuticals that are highly prescribed in the United States and other chemicals like bis-phenol A (BPA) in plastics, phthalates in cosmetics and teratogenic chemicals [1].

Psychoactive pharmaceuticals like fluoxetine, venlafaxine and carbamazepine, have been detected in the drinking water at very low concentrations $[2,6,7]$. These pharmaceuticals, some of which are metabolically active and have relatively long half-lives for over a month, reach wastewater treatment plants (WWTP) through excretion by clinical patients $[7,8]$. Due to the chemical properties of these drugs and inefficient filtration of WWTP, these drugs end up mixing up with the ground water, and thus reach drinking water at low concentrations $[7,8]$.

We previously hypothesized that psychoactive pharmaceuticals as environmental contaminants alter neuronal gene expression associated with neurological disorders like ASD. To determine this, our lab treated juvenile fathead minnows (Pimephales promelas) with psychoactive pharmaceuticals (fluoxetine, venlafaxine and carbamazepine) individually and in mixtures at environmentally relevant concentrations $[2,6]$. After treating them for 15 days, we extracted the brains and carried out microarray analysis. Using gene set enrichment analysis (GSEA) [9], we identified enrichment (up- or down-regulation) of gene sets associated with neuronal growth, regulation and development in the juvenile minnow brains in response to psychoactive drug exposure [2, 6]. We also identified altered neuronal gene sets associated with neurological disorders, including ASD [6]. Moreover, fish exposed to psychoactive pharmaceuticals exposed had an altered behavioral phenotype $[2,10]$.

In the present study, we hypothesized that psychoactive pharmaceuticals (fluoxetine, venlafaxine and carbamazepine) at environmental concentrations would alter in vitro human neuronal gene expression that is 1) similar to gene expression profile in fathead minnow, and 2) associated with neuro-developmental disorders. To determine if altered gene expression profile was associated with idiopathic autism, we also treated human neuronal cells with valproic acid, which is known to induce autism-like phenotypes in mice [11]. Identifying altered gene expression profiles in treated cells and finding a similar pattern with valproic acid induced gene expression would reveal the extent to which psychoactive pharmaceuticals at very low concentrations could induce gene expression associated with potential neurological disorders like ASD.

\section{Methods \\ Cell culture and differentiation}

Human SK-N-SH cell line was obtained from American Type Culture Collection (ATCC \#HTB-11). Cells were cultured in Eagle's Minimum Essential Medium (EMEM; ATCC). This media was supplemented with $1 \%$ penicillinstreptomycin-neomycin (Sigma) and $10 \%$ (v/v) fetal bovine serum (FBS). Retinoic acid (RA; Sigma) was used to induce SK-N-SH cells [12] to differentiate into more neuron-like cells [13] because this cell line is a mixture of different cell types. Approximately 15,000 cells were cultured in T-75 flask (Corning) supplemented with EMEM media for two days, and then Retinoic acid $(10 \mu \mathrm{M})$ was added. Cells were treated with RA for two weeks and media was replaced every three-four days [12]. Cultures were monitored visually using light microscopy for morphological changes, and evaluated for neuronal cell markers (NeuN, PSD95 and NCAM) during the differentiation process $[12,14]$.

\section{Pharmaceuticals treatments}

Stock solutions (10 mM) of fluoxetine (FLX; Sigma F133; Active metabolite), venlafaxine (VNX; Sigma D2069; Active metabolite) and carbamazepine (CBZ; Sigma C4206; Active metabolite), and $1 \mathrm{mM}$ stock solution of valproic acid (VPA; Santa Cruz Biotechnology sc211393; Active metabolite) were prepared in dimethyl sulfoxide (DMSO). After differentiation for two weeks, cells were treated with a mixture composed of (MIX: FLX $10 \mu \mathrm{g} / \mathrm{l}$; VNX $50 \mu \mathrm{g} / \mathrm{l}$; CBZ $100 \mu \mathrm{g} / \mathrm{l}$ ) [2], and Valproate (VPA: $4.9 \mathrm{mg} / \mathrm{l}$ ) [15]. Control cells were treated with DMSO (vehicle) only, and the final concentration of DMSO in the cultures was $0.05 \%$. To examine if psychoactive pharmaceuticals in drinking water at environmental concentrations could induce neuronal gene expression, we chose to treat neuronal cells with the mixture of three pharmaceuticals (fluoxetine, carbamazepine and venlafaxine). Cells were treated in three replicates with the pharmaceuticals for $48 \mathrm{~h}$ in EMEM media without FBS to avoid any binding of pharmaceuticals with the serum proteins. All of the treatments were shown not to affect overall cell viability with respect to control (no treatment), based on the adherent nature of the monolayers and the result from CyQuant Viability Assay [16]. After treating cells for $48 \mathrm{~h}$, they were collected with Versene solution (Gibco).

\section{RNA extraction, cDNA synthesis and sequencing}

After 48 h of exposure, cells were collected with trypsin, centrifuged, and RNA was extracted using Qiagen RNeasy Plus Mini Kit (74134) according to the manufacturer's 
protocol. Total RNA concentration was determined using NanoDrop (Thermo Scientific), and the RNA integrity value (RIN) was analyzed on Agilent 2100 Bioanalyzer (Agilent, Santa Clara, CA). RNA was quantified with Qubit spectrophotometer (Life technologies). Using Illumina Tru-seq stranded total RNA kit, cDNA library was prepared in following steps: RiboZero depletion and fragmentation, first and second strand cDNA synthesis, adenylation of 3 ' ends, adapter ligation, PCR amplification, library validation using qPCR - Kapa Biosystems Library Quantification Kit, and normalization and pooling in preparation for cluster generation on MiSeq. Samples were then loaded onto flow cell (MiSeq Reagent kit v3 150 cycle) and sequenced on Illumina MiSeq 2 according to the manufacturer's instructions.

\section{Bioinformatics data analysis}

\section{Quality control, alignment, and read counting}

In total, three treatments (mixture, valproate, and control) with three replicates each were sequenced with five flow cells using Illumina MiSeq, which generated more than $10 \mathrm{M}$ paired-end reads for each replicate (refer Additional file 1). The raw sequences in FASTQ files underwent quality control analysis using FastQC (http:// www.bioinformatics.babraham.ac.uk/projects/fastqc). All the samples were sequenced at $75 \mathrm{~b}$ read length and they all passed the quality check. We aligned the quality checked reads to human genome hg19 using TopHat version 2.0.11 [17]. Most of the reads ( 90 \%) had average base quality above Q30. The reference genome sequence, the bowtie index files for the reference genome sequence, and the gene annotation file were downloaded from Illumina iGenomes project (http://support.illumina. $\mathrm{com} /$ sequencing/sequencing_software/igenome.html). The number of reads that map to human known genes were counted by summerizeOverlaps function in GenomicAlignments $\mathrm{R}$ package [18]. Only the genes that have at least one read for all replicates were retained for downstream analysis.

\section{Analysis of differential gene expression}

Differentially expressed genes were identified using DESeq2 version 1.6.1 [19]. Differentially expressed genes from 2 groups (Mixture and Valproate) with respect to the control treatment were identified.

\section{Gene set enrichment analysis}

We used GAGE version 2.14.4 [20] for gene set enrichment analysis. We used GAGE to analyze gene sets that were analyzed previously in Fish brains $[2,6]$. These gene sets are grouped as Neuronal development, regulation, growth; Neurological Disorders (ND); Autism spectrum disorders (ASD) $[2,6]$. For extensive analysis, we also analyzed gene sets from MSigDB 'C2' and 'C5' (refer
Additional file 2). Enrichment analyses were carried out using gage function using non-parametric KolmogorovSmirnov tests. We used GAGE package to identify significantly enriched (significantly up- and/or down-regulated; $P$-value $<0.01$ and $Q$-value $<0.1)$ gene sets within mixture and valproate treatments.

\section{Results}

Patterns of psychoactive pharmaceuticals - induced in vitro gene expression in neuronal development, growth and regulation

We postulated that psychoactive pharmaceuticals at environmental concentrations would alter gene expression of neuronal systems. Support for this hypothesis would suggest that dysregulation of neuronal systems would result in altered neuronal circuits and may result in fewer neuronal connections. To address this question we used differentiated SK-N-SH neuroblastoma cells and treated them with the mixture (MIX: FLX-10 $\mu \mathrm{g} / \mathrm{l} ; \mathrm{VNX}-50 \mu \mathrm{g} / \mathrm{l}$; CBZ-100 $\mu \mathrm{g} / \mathrm{l}$ ), and valproate (VPA: $4.9 \mathrm{mg} / \mathrm{l}$ ) in replicates of three samples for each treatment.

For the development collection, we observed six enriched gene sets (significantly down-regulated, $P$-value $<0.01, Q$ value $<0.1)$ by MIX treatment. VPA treatment enriched the expression of seven gene sets (four gene sets downand three gene sets up-regulated, Additional file 3). AXONOGENESIS, REGULATION OF NEUROGENESIS and SYNAPSE PART gene sets were enriched in both VPA and MIX treatments. And, SYNAPSE PART gene set was also enriched (up-regulated) in fish brains.

For the regulation collection, we observed 14 enriched gene sets (13 gene sets down- and one gene set upregulated, $P$-value $<0.01, Q$-value $<0.1$ ) by MIX treatment. VPA treatment enriched the expression of eight gene sets (up-regulated, Additional file 3). All eight up-regulated gene sets in VPA treatment were down-regulated in MIX treatment. Among those sets, two gene sets (NEUROTRANSMITTER_BINDING and SYNAPSE) were also enriched in fish brains.

For the growth collection, we observed eight enriched gene sets (all down-regulated, $P$-value $<0.01, Q$-value $<0.1$ ) by MIX treatment. VPA treatment enriched the expression of five gene sets (up-regulated, Additional file 3). All five up-regulated gene sets in VPA treatment were downregulated in MIX treatment. Two gene sets (AXON and NEURON_PROJECTION) were also enriched in fish brains.

Patterns of psychoactive pharmaceuticals - induced in vitro gene expression in neurological disorders (ND) and ASD groups

We then sought to determine if altered in vitro gene expression was associated with neurological disorders and ASD. To accomplish this, we analyzed and compared 
the gene expression of already published ND and ASD gene sets in MIX and VPA treatments.

For the ND collection, we observed six enriched gene sets (four down- and two up-regulated, $P$-value $<0.01$, $Q$-value $<0.1)$ by MIX treatment. VPA treatment enriched the expression of three gene sets (two gene sets downand one gene set up-regulated, Additional file 3). AUTISM_IDIOPATHIC and PARKINSONS gene sets were enriched in both VPA and MIX treatments as well as in fish brains.

For the ASD collection, we observed two enriched gene sets (both up-regulated, $P$-value $<0.01, Q$-value $<0.1$ ) by MIX treatment. VPA treatment enriched the expression of five gene sets (down-regulated, Additional file 3). ASD_MILD gene set was enriched in both VPA and MIX treatments as well as in fish brains.

\section{Ranked gene lists from the mixture and valproate treatment}

We sorted all genes from gene expression profiles in human neuronal cells treated with the MIX (FLX, VNX, CBZ) and valproate (VPA). In each treatment, we sequenced 17,000 genes and analyzed their expression. We then ranked them based on their fold change expression within each treatment. Tables 1 and 2 show, the 50 most strongly up-and down-regulated genes. We also reported the fold-change score of genes which were presented on the fish microarray chip.

\section{Discussion}

\section{Comparison between human MIX treatment and fish gene expression patterns}

The results partially supported our first hypothesis that the MIX treatment on human SK-N-SH cell line induced gene sets enrichment patterns similar to that of the fish experiment following mixture treatment, although the degree of similarity was not high.

Among the neural circuit development gene sets, all the six significantly enriched gene sets in human neuronal cells following MIX treatment were enriched in a downregulated manner; whereas in the fish experiment, the two significantly enriched sets were both up-regulated. We do not know why the direction was opposite, but we do notice that, one gene set, SYNAPSE PART, was enriched in both treatments [2]. Previous studies have found that altered expression of NCAM, IRX3 and NKX6.1 genes in SYNAPSE PART change the fate and position of neurons generated in the chick neural tube [21, 22]. Also within those down-regulated gene sets contains gene PSD95 (DLG4) and GABA, which have recently been found to be associated with neurological disorders like autism by altering synaptic assembly [14, 23, 24].

In the growth group, we observed similar patterns where all enriched sets in MIX treatment were down- regulated and all those in the fish experiment were upregulated. Among those sets, two were enriched in both treatments, AXON and NEURON PROJECTION. Other studies have found that genes within these two gene sets modulate the fate, lineage, and timing of neuronal development by playing a critical role in the formation and maturation of neural circuits [23, 25, 26].

In the regulation group, we observed that NEUROTRANSMITTER BINDING gene set was down-regulated in fish brains as well as in human cells [2]. This could be possible due to the therapeutic effect of fluoxetine (SSRI) in the mixture treatment [2]. Fluoxetine is known to reduce the re-uptake of serotonin by inhibiting monoamine transporters on the pre-synaptic neuronal membrane $[27,28]$. Due to the longer availability of neurotransmitters in the synaptic cleft, the expression of serotonin receptors is down-regulated, thus a decrease in neurotransmitter binding [29]. Another gene set SYNAPSE was significantly up-regulated in fish brains [2], but down-regulated in treated human cells. This gene set was responsible for modulating wiring of neuronal circuits by controlling the number of synapse as well as organization of synaptic assembly and specificity [26, 30]. Altered synaptogenesis has been strongly considered as a potential mechanism in ASD pathogenesis [31, 32].

\section{Comparison between human MIX and VPA gene expression patterns}

We used valproate (an anticonvulsant) to treat human neuronal cells as a positive control because prenatal exposure of valproate has been found to be strongly associated with autism [3] and valproate is known to induce autism-like phenotypes in mice [11]. Similarly, carbamazepine (presented in the mixture treatment) is a mood stabilizer and anticonvulsant $[11,33]$ and it also inhibits the epileptic effects in the brain by blocking sodium channels $[11,33]$. By and large, the results support our second hypothesis that the MIX and VPA treatments change the RNA expression profile in similar ways, albeit in an interesting fashion, since the two treatments often enrich the same gene sets but in different directions.

In the development group of VPA treatment, we found four gene sets significantly down-regulated and three gene sets significantly up-regulated in human neuronal cells. Three gene sets (AXONOGENESIS, REGULATION OF NEUROGENESIS and SYNAPSE PART) were enriched in both mixture and valproate treatments, but in the opposite direction. Similar to fish gene expression, VPA treatment up-regulated SYNAPSE PART gene set [2]. This states that VPA exposure might be associated with disturbed neuronal fate and position [21, 22] as well as synaptic assembly [1, 22, 34].

In the growth group, we observed that five gene sets were up-regulated in the VPA treatment. Interestingly, 
Table 1 Ranked gene list from the mixture treatment (50 most up- and down-regulated)

\begin{tabular}{|c|c|c|c|c|c|c|c|c|c|}
\hline \multicolumn{5}{|c|}{ Up-regulated genes } & \multicolumn{5}{|c|}{ Down-regulated genes } \\
\hline Rank & Genes & Fold change & $P$-value & Fold change in fish brains & Rank & Genes & Fold change & $P$-value & Fold change in fish brains \\
\hline 1 & FAM129A & 3.25 & 0.000 & & 17350 & ANGPTL4 & -3.36 & 0.000 & \\
\hline 2 & CACNA2D3 & 1.99 & 0.000 & 0.90 & 17349 & THBS1 & -2.19 & 0.000 & 0.34 \\
\hline 3 & NUPR1 & 1.97 & 0.002 & -0.10 & 17348 & STARD13 & -2.17 & 0.000 & -0.46 \\
\hline 4 & GRM8 & 1.90 & 0.000 & & 17347 & $D R P 2$ & -2.15 & 0.000 & \\
\hline 5 & PTCHD1 & 1.89 & 0.000 & & 17346 & TNNT2 & -2.11 & 0.000 & -0.23 \\
\hline 6 & TMEM132C & 1.76 & 0.000 & & 17345 & $A Q P 10$ & -2.10 & 0.000 & \\
\hline 7 & DD/T3 & 1.55 & 0.000 & 0.73 & 17344 & STRA6 & -1.97 & 0.000 & -0.10 \\
\hline 8 & XKR4 & 1.53 & 0.010 & & 17343 & PAPPA2 & -1.92 & 0.000 & \\
\hline 9 & CHRNA10 & 1.33 & 0.002 & & 17342 & NEDD9 & -1.88 & 0.000 & -1.21 \\
\hline 10 & LPHN3 & 1.24 & 0.004 & & 17341 & GGTA1 & -1.85 & 0.001 & \\
\hline 11 & SERPINF2 & 1.23 & 0.008 & 0.15 & 17340 & SH3TC1 & -1.83 & 0.000 & \\
\hline 12 & NKX3-1 & 1.22 & 0.008 & & 17339 & ADAMTSL2 & -1.80 & 0.000 & -0.25 \\
\hline 13 & HRK & 1.22 & 0.051 & & 17338 & RGS13 & -1.78 & 0.000 & \\
\hline 14 & SCARNA2O & 1.22 & 0.009 & & 17337 & ANO1 & -1.77 & 0.000 & \\
\hline 15 & PABPC3 & 1.21 & 0.006 & & 17336 & LOC283731 & -1.75 & 0.000 & \\
\hline 16 & KIT & 1.18 & 0.001 & 0.15 & 17335 & $C M K L R 1$ & -1.73 & 0.000 & 0.86 \\
\hline 17 & KIAA1045 & 1.18 & 0.002 & -0.12 & 17334 & $V I P$ & -1.71 & 0.000 & \\
\hline 18 & DLL1 & 1.15 & 0.013 & 0.05 & 17333 & HTR2B & -1.69 & 0.000 & \\
\hline 19 & C9orf150 & 1.12 & 0.000 & & 17332 & RAG2 & -1.67 & 0.004 & 0.20 \\
\hline 20 & SNORA14A & 1.11 & 0.164 & & 17331 & ACVRL1 & -1.67 & 0.000 & \\
\hline 21 & B3GALT1 & 1.09 & 0.134 & & 17330 & VCAN & -1.66 & 0.000 & \\
\hline 22 & RIMBP3C & 1.09 & 0.096 & & 17329 & C6 & -1.65 & 0.006 & -1.11 \\
\hline 23 & NOSIAP & 1.06 & 0.172 & 0.37 & 17328 & ENO3 & -1.64 & 0.000 & -0.00 \\
\hline 24 & GLYATL2 & 1.06 & 0.021 & & 17327 & TM4SF1 & -1.63 & 0.008 & 0.22 \\
\hline 25 & STAC2 & 1.04 & 0.259 & & 17326 & PCTK3 & -1.62 & 0.004 & \\
\hline 26 & CRYBB2 & 1.04 & 0.020 & 0.01 & 17325 & FAM65C & -1.60 & 0.000 & \\
\hline 27 & SLC16A10 & 1.01 & 0.167 & -0.31 & 17324 & FL36208 & -1.60 & 0.000 & \\
\hline 28 & $\mathrm{CDH7}$ & 1.01 & 0.031 & -0.00 & 17323 & LOC400950 & -1.58 & 0.000 & \\
\hline 29 & MYB & 1.01 & 0.030 & -0.17 & 17322 & RARRES3 & -1.58 & 0.000 & \\
\hline 30 & LCA5L & 1.00 & 0.176 & & 17321 & SLCO4C1 & -1.53 & 0.000 & \\
\hline 31 & ID4 & 0.99 & 0.033 & -0.17 & 17320 & PRAP1 & -1.52 & 0.022 & \\
\hline 32 & MALAT1 & 0.99 & 0.004 & & 17319 & SLC18A1 & -1.51 & 0.000 & \\
\hline 33 & SNAR-A3 & 0.99 & 0.230 & & 17318 & TFPI2 & -1.51 & 0.000 & \\
\hline 34 & EDN1 & 0.98 & 0.033 & & 17317 & FBP1 & -1.50 & 0.000 & 0.43 \\
\hline 35 & VSTM2A & 0.97 & 0.000 & & 17316 & FGL1 & -1.49 & 0.001 & \\
\hline 36 & HERV-FRD & 0.97 & 0.036 & & 17315 & COL9A3 & -1.48 & 0.001 & \\
\hline 37 & CNTD1 & 0.97 & 0.037 & -1.03 & 17314 & LRRTM1 & -1.48 & 0.015 & 0.25 \\
\hline 38 & ELL2 & 0.96 & 0.000 & -0.30 & 17313 & BTK & -1.48 & 0.000 & -0.30 \\
\hline 39 & SLC13A3 & 0.96 & 0.027 & 0.02 & 17312 & $S L C 24 A 2$ & -1.46 & 0.000 & 0.11 \\
\hline 40 & DENNDIB & 0.96 & 0.114 & 0.06 & 17311 & IRF6 & -1.45 & 0.000 & 0.17 \\
\hline 41 & CYP2C18 & 0.95 & 0.041 & & 17310 & LOC392232 & -1.45 & 0.012 & \\
\hline 42 & $A M N$ & 0.95 & 0.041 & -0.33 & 17309 & RD3 & -1.45 & 0.000 & \\
\hline
\end{tabular}


Table 1 Ranked gene list from the mixture treatment (50 most up- and down-regulated) (Continued)

\begin{tabular}{llllllllll}
\hline 43 & NCRNA00087 & 0.95 & 0.301 & & 17308 & DDC & -1.43 & 0.000 & \\
44 & FUT9 & 0.94 & 0.042 & -0.19 & 17307 & PRLHR & -1.43 & 0.008 & \\
45 & SCGB1D2 & 0.92 & 0.047 & & 17306 & NOTUM & -1.42 & 0.052 & -0.31 \\
46 & TRIB3 & 0.92 & 0.000 & 0.67 & 17305 & TMIGD2 & -1.41 & 0.008 & \\
47 & MYBL1 & 0.91 & 0.019 & & 17304 & RTL1 & -1.40 & 0.048 & -0.40 \\
48 & ZNF726 & 0.91 & 0.260 & & 17303 & P2RX6 & -1.40 & 0.000 & \\
49 & DLEU1 & 0.90 & 0.173 & & 17302 & DKK1 & -1.39 & 0.000 & \\
50 & SLC7A11 & 0.90 & 0.309 & -0.41 & 17301 & TRIM9 & -1.38 & 0.000 & \\
\hline
\end{tabular}

Table representing the list of 50 most up- and down-regulated genes in human neuronal cells treated with the mixture (FLX, VNX, CBZ). Genes were ranked based on their expression in fold-change in human cells. P-value represents the significance level of the expression change in the mixture treatment than control. Corresponding fold change of genes in fish brains is also reported in this table, where blank cells represents genes that are not found in the fish microarray chip

the same five sets were all down-regulated in the MIX treatment. This suggested that both treatments disturbed the human gene expression in similar pathways despite the different directions. One gene set NEURON PROJECTION was up-regulated similar to fish brains [2]. In the regulation group, we noticed similar results. Eight gene sets were up-regulated in valproate treatment, but down-regulated in the mixture treatment. From this repeating phenomenon of opposite direction enrichment of the same gene set, we postulate a general pattern that the three pharmaceuticals (fluoxetine, venlafaxine and carbamazepine) and valproate dysregulate the same pathways in different directions. However, more investigation is needed to confirm or disprove this postulation. We also plotted multi-dimensional scaling using edgeR [35] and observed that mixture treatment samples were somewhat closer to valproate samples than control samples (refer Additional file 1). However, MIX and VPA samples were still apart, confirming that these two treatments did not exhibit similar response.

\section{Association of human MIX and VPA gene expression patterns with neurological disorders}

To determine the extent to which altered gene expression in both MIX and VPA treatments were associated with neurological disorders (including ASD), we examined the expression of the already-published gene sets from neurological disorders (ND) and ASD groups in both treatments. We also compared these to their corresponding expression pattern in fish brains. For ND group, MIX and VPA treatments altered PARKINSONS and Autism_Idiopathic gene sets significantly but in different directions. Interestingly, mixture treatment of human cells and fish brains up-regulated Autism_Idiopathic gene set [6]. For another ASD group, MIX treatment upregulated two gene sets (ASD_2Class and ASD_Mild) similar to fish brains [6]. On the other side, VPA treatment down-regulated three gene sets (ASD_2Class, ASD_Mild, ASD_Savant) in a different direction compared to the expression in fish brains [6]. These expression patterns stated that VPA and MIX treatments of human cells exhibited a similar response to neurological disorders (including ASD), suggesting a common induction effect.

\section{Insights into important genes in both MIX and VPA treatments}

We sought to identify important, or novel genes that were significantly up- and down-regulated in human neuronal cells treated with MIX and VPA. We generated ranked lists of genes based on their fold change, and tabulated the most 50 strongly up- and downregulated genes (Table 1 and 2). We also compared genes from ranked lists with the ones from fish microarray data. For MIX treatment, we noticed four genes (NUPR1, RTL1, THBS1 and HTR2B) which could be considered important and novel. The thrombospondin (THBS1) gene plays an important role in synaptogenesis in the developing brain [36]. Recent association studies have found that both rare and common variants of this gene are associated with autism [36]. Although this gene was found to be down-regulated by two-fold, it was up-regulated in fish brains under similar mixture treatment [2]. Another important gene, $H T R 2 B$, which codes for serotonin receptor $2 \mathrm{~B}$, was down-regulated by $\sim$ two-fold in human cells. Similar serotonin receptor genes were also found to be down-regulated in fish brains. Moreover, recent protein studies in our lab showed that HTR2B protein was down-regulated in the same mixture (FLX, VNX, CBZ) treatment. This mechanism is explained by the drug effect of fluoxetine (SSRI), which provides more neurotransmitter in the synaptic cleft, thus reducing serotonin receptors [29].

In the ranked list of genes by valproate (VPA) treatment, we found three genes (VSNL1, PTER and OXTR) of particular importance. VSNL1 gene, which encodes for visinin-like protein 1 in humans, modulates neuronal morphology by controlling the key signaling pathways in 
Table 2 Ranked gene list from the valproate treatment (50 most up- and down-regulated)

\begin{tabular}{|c|c|c|c|c|c|c|c|c|c|}
\hline \multicolumn{5}{|c|}{ Up-regulated genes } & \multicolumn{5}{|c|}{ Down-regulated genes } \\
\hline Rank & Genes & Fold change & $P$-value & Fold change in fish brains & Rank & Genes & Fold change & $P$-value & Fold change in fish brains \\
\hline 1 & OPRK1 & 4.03 & 0.000 & -0.29 & 17673 & CMKLR1 & -2.81 & 0.000 & 0.86 \\
\hline 2 & NEUROG2 & 3.55 & 0.000 & & 17672 & ADAMTS2 & -2.74 & 0.000 & 0.09 \\
\hline 3 & VSNL1 & 3.19 & 0.000 & 2.84 & 17671 & HIST1H4L & -2.67 & 0.000 & \\
\hline 4 & GALNAC4S-6ST & 3.09 & 0.000 & 0.96 & 17670 & ELFN1 & -2.44 & 0.000 & \\
\hline 5 & POSTN & 2.93 & 0.000 & 1.22 & 17669 & COL4A2 & -2.39 & 0.000 & -0.42 \\
\hline 6 & EVX2 & 2.86 & 0.000 & & 17668 & GREM2 & -2.33 & 0.000 & \\
\hline 7 & SERPINE1 & 2.69 & 0.000 & -1.45 & 17667 & FLJ45455 & -2.32 & 0.000 & 0.86 \\
\hline 8 & ODZ1 & 2.64 & 0.000 & & 17666 & VCAN & -2.31 & 0.000 & \\
\hline 9 & DRD5 & 2.34 & 0.000 & -0.23 & 17665 & sOCS3 & -2.29 & 0.000 & 0.02 \\
\hline 10 & CSGALNACT1 & 2.33 & 0.001 & & 17664 & TGFBI & -2.27 & 0.000 & -1.11 \\
\hline 11 & HOXD13 & 2.31 & 0.000 & & 17663 & C11orf53 & -2.26 & 0.000 & \\
\hline 12 & C3orf57 & 2.29 & 0.000 & & 17662 & SNAl1 & -2.17 & 0.000 & -0.82 \\
\hline 13 & B3GALT1 & 2.29 & 0.000 & & 17661 & LOC283480 & -2.11 & 0.000 & \\
\hline 14 & SECTMI & 2.28 & 0.001 & & 17660 & $\mid D 1$ & -2.05 & 0.000 & 0.23 \\
\hline 15 & PLAU & 2.20 & 0.001 & & 17659 & PPP1R9A & -2.03 & 0.000 & -0.05 \\
\hline 16 & PDEIA & 2.19 & 0.000 & & 17658 & LOC646498 & -2.02 & 0.004 & \\
\hline 17 & VIM & 2.14 & 0.000 & 0.25 & 17657 & RPS16P5 & -2.02 & 0.000 & \\
\hline 18 & MME & 2.11 & 0.000 & & 17656 & COL4A1 & -1.98 & 0.000 & 0.10 \\
\hline 19 & KCNJ2 & 2.09 & 0.000 & & 17655 & GLT8D2 & -1.98 & 0.000 & \\
\hline 20 & HS6ST2 & 2.09 & 0.000 & -0.15 & 17654 & CYP26A1 & -1.96 & 0.000 & 0.26 \\
\hline 21 & SNORA42 & 2.00 & 0.003 & & 17653 & SLC10A1 & -1.95 & 0.004 & \\
\hline 22 & RASEF & 1.99 & 0.000 & 0.31 & 17652 & PART1 & -1.94 & 0.000 & \\
\hline 23 & APOL6 & 1.93 & 0.000 & & 17651 & OXTR & -1.89 & 0.000 & 1.26 \\
\hline 24 & NCRNA00164 & 1.91 & 0.007 & & 17650 & PTPLAD2 & -1.84 & 0.007 & \\
\hline 25 & COLQ & 1.91 & 0.000 & & 17649 & C9orf131 & -1.83 & 0.001 & \\
\hline 26 & CACNA2D3 & 1.90 & 0.000 & 0.90 & 17648 & FOS & -1.80 & 0.000 & -0.63 \\
\hline 27 & DEGS2 & 1.90 & 0.000 & & 17647 & C9orf135 & -1.79 & 0.009 & \\
\hline 28 & NHS & 1.89 & 0.000 & & 17646 & GLP1R & -1.79 & 0.000 & \\
\hline 29 & PTER & 1.89 & 0.001 & 0.11 & 17645 & ETS1 & -1.79 & 0.000 & -0.79 \\
\hline 30 & EDIL3 & 1.87 & 0.000 & -0.41 & 17644 & $\mathrm{NXPH3}$ & -1.79 & 0.000 & \\
\hline 31 & INSM2 & 1.85 & 0.000 & & 17643 & LOC100128505 & -1.79 & 0.013 & \\
\hline 32 & GFRA3 & 1.82 & 0.000 & & 17642 & KCTD12 & -1.76 & 0.000 & 0.49 \\
\hline 33 & PROKR2 & 1.82 & 0.000 & & 17641 & ANGPTL4 & -1.74 & 0.000 & \\
\hline 34 & GRM8 & 1.81 & 0.001 & & 17640 & FOSB & -1.73 & 0.000 & \\
\hline 35 & TLE4 & 1.79 & 0.000 & & 17639 & MPPED2 & -1.72 & 0.000 & -0.62 \\
\hline 36 & WNT5A & 1.77 & 0.001 & -0.38 & 17638 & C11orf92 & -1.71 & 0.003 & \\
\hline 37 & SMOC2 & 1.77 & 0.000 & -0.26 & 17637 & WDR38 & -1.69 & 0.001 & \\
\hline 38 & VCAM1 & 1.76 & 0.014 & 0.53 & 17636 & GALNT14 & -1.68 & 0.000 & 0.03 \\
\hline 39 & FRMD $4 B$ & 1.74 & 0.000 & 0.10 & 17635 & LOC283143 & -1.67 & 0.002 & \\
\hline 40 & PTRF & 1.74 & 0.000 & & 17634 & ACCN1 & -1.66 & 0.002 & \\
\hline 41 & $D L K 1$ & 1.70 & 0.000 & 0.69 & 17633 & C14orf53 & -1.66 & 0.002 & \\
\hline 42 & PTCHD1 & 1.67 & 0.001 & & 17632 & TAPBPL & -1.65 & 0.021 & \\
\hline
\end{tabular}


Table 2 Ranked gene list from the valproate treatment (50 most up- and down-regulated) (Continued)

\begin{tabular}{|c|c|c|c|c|c|c|c|c|c|}
\hline 43 & GPR126 & 1.66 & 0.022 & & 17631 & NRP1 & -1.63 & 0.000 & 1.12 \\
\hline 44 & ANKDD1B & 1.65 & 0.000 & & 17630 & BFSP2 & -1.63 & 0.002 & \\
\hline 45 & MOXD1 & 1.65 & 0.002 & -1.00 & 17629 & SMAD7 & -1.63 & 0.000 & 0.08 \\
\hline 46 & FAM $111 B$ & 1.65 & 0.000 & & 17628 & HOPX & -1.62 & 0.028 & \\
\hline 47 & LOC728739 & 1.63 & 0.002 & & 17627 & ERBB4 & -1.62 & 0.000 & \\
\hline 48 & CHRNA10 & 1.62 & 0.002 & & 17626 & PAPPA2 & -1.62 & 0.000 & \\
\hline 49 & NELL 1 & 1.62 & 0.000 & & 17625 & PCDHGB8P & -1.62 & 0.011 & \\
\hline 50 & NELL2 & 1.62 & 0.000 & -0.76 & 17624 & LOC284454 & -1.60 & 0.000 & \\
\hline
\end{tabular}

Table representing the list of 50 most up- and down-regulated genes in human neuronal cells treated with the valproate. Genes were ranked based on their expression in fold- change in human cells. $P$-value represents the significance level of the expression change in the mixture treatment than control. Corresponding fold change of genes in fish brains is also reported in this table, where blank cells represents genes that are not found in the fish microarray chip

CNS. This gene was up-regulated by three-fold in the valproate treatment. We observed similar up-regulation of this gene in fish brains following mixture (FLX, VNX, CBZ) treatment. Other studies have recently found the association of single-nucleotide polymorphisms (SNPs) in the VSNL1 gene with neurological disorders like schizophrenia [37]. Another important gene, oxytocin receptor $(O X T R)$ was found to be down-regulated by two-fold in human cells treated with valproate (Table 2 ). However, this gene was up-regulated in fish brains exposed to the mixture of psychoactive pharmaceuticals [2]. Moreover, recent protein studies in our lab showed an increased expression of OXTR in the same cells treated with carbamazepine and the mixture (FLX, VNX, CBZ). Other studies have shown that OXTR serves as an anxiolytic agent by modulating serotonin release in serotonergic neurons of the raphe nuclei [38].

\section{Conclusions}

To investigate the environmental trigger for idiopathic autism, we focused on psychoactive pharmaceuticals, potential environmental contaminants that have been detected in drinking water. We found that psychoactive pharmaceuticals altered the gene expression of neuronal systems in vitro at environmental concentrations. These altered gene expressions are associated with potential neurological disorders by playing a key role in the formation, growth and regulation of neurons. Our data suggests that psychoactive pharmaceuticals might disrupt neuronal connections by altering the gene expression associated with neuronal growth, development and regulation.

\section{Additional files}

Additional file 1: R-programming code for RNA-Seq analysis and multidimensional scaling (MDS) function. The file contains R-code for analysis of RNA-Seq data for mixture and valproate treatments. This file also includes the code for plotMDS function for using multi-dimensional scaling. (DOCX $61 \mathrm{~kb}$ )
Additional file 2: Complete data of gene sets analyzed. Excel file containing a complete data of all gene sets (neuronal development, growth, regulation; neurological disorders and ASD group) that were enriched (upand down-regulated) in mixture (carbamazepine, fluoxetine and venlafaxine) and valproate treatments. We also reported $P$-values and $Q$ values for individual gene sets. (XLSX $1621 \mathrm{~kb}$ )

Additional file 3: Descriptions of gene sets and results of analyses. This file contains table that represents the analyses of gene sets within neuronal systems (a. Development, b. Regulation, c. Growth), (d) Neurological Disorders, and (e) ASD groups. Source indicates the database from where the gene set was derived, Gene Ontology (GO) or Molecular Signatures Database (MSigDB). Size represents the number of genes in each gene set. MIX_UP, MIX_DOWN, VPA_UP and VPA_DOWN indicates gene sets which were found up-regulated in the mixture, down-regulated in the mixture, up-regulated in the valproate, and down-regulated in the valproate treatments, respectively. Gene sets with $P$-value $<0.01$ and $Q$-value $<0.1$ were considered as statistically significantly enriched. For non-significant gene sets, refer Additional file 2 for $P$-values and Q-values. Enriched gene sets in human cells and corresponding scores are marked in bolds. Enriched gene sets in fish brains are italicized and marked in parentheses. (DOCX $124 \mathrm{~kb}$ )

\section{Acknowledgements}

The authors thank Late Dr. Chris Cretekos for providing the guidance for using neuronal cells for our study. We also thank Dr. Jean Pfau for providing her laboratory space and reagents for neuronal cell culture.

\section{Declarations}

The publication costs for this article were funded by an Institutional Development Award (IDeA) from the National Institute of General Medical Sciences of the National Institutes of Health under Grant \#P20GM103408. This article has been published as part of BMC Genomics Volume 17 Supplement 3, 2016: Selected articles from the 12th Annual Biotechnology and Bioinformatics Symposium: genomics. The full contents of the supplement are available online at https://bmcgenomics.biomedcentral.com/ articles/supplements/volume-17-supplement-3.

\section{Availability of data and materials}

The RNA-Seq dataset for this research article is available in the Gene Expression Omnibus (GEO) repository, [GSE80635 and http://www.ncbi.nlm.nih.gov/geo/ query/acc.cgi?acc=GSE80635].

\section{Authors' contributions}

GK, YX and MAT designed the experiments, and GK performed them. MAT provided the direction and guidance for the research. YX and LY carried out analyses using R-programming and GK wrote the manuscript. All authors have read and approved the final manuscript.

\section{Competing interests}

The authors declare that they have no competing interests. 


\section{Consent for publication}

Not applicable.

\section{Ethics approval and consent to participate}

Not applicable.

\section{Author details}

'Department of Biological Sciences, Idaho State University, Stop 8007, 9215 8th Ave, Pocatello, ID 83209-8007, USA. ${ }^{2}$ Department of Medical Pathology and Laboratory Medicine, University of California at Davis, Davis, CA 95817, USA. ${ }^{3}$ Present Address: Institute for Pediatric Regenerative Medicine, Shriners Hospitals for Children, Northern California, 2425 Stockton Boulevard, Sacramento, CA 95817, USA. ${ }^{4}$ Division of Biological Sciences, University of Montana, 32 Campus Dr, Missoula, MT 59812, USA.

\section{Published: 29 June 2016}

\section{References}

1. Landrigan PJ. What causes autism? Exploring the environmental contribution. Curr Opin Pediatr. 2010;22(2):219-25.

2. Thomas MA, Joshi PP, Klaper RD. Gene-class analysis of expression patterns induced by psychoactive pharmaceutical exposure in fathead minnow (Pimephales promelas) indicates induction of neuronal systems. Comp Biochem Physiol C-Toxicol Pharmacol. 2012;155(1):109-20

3. Dufour-Rainfray D, Vourc'h P, Tourlet S, Guilloteau D, Chalon S, Andres CR. Fetal exposure to teratogens: Evidence of genes involved in autism. Neurosci Biobehav Rev. 2011;35(5):1254-65.

4. State MW, Levitt P. The conundrums of understanding genetic risks for autism spectrum disorders. Nat Neurosci. 2011;14(12):1499-506.

5. Kaushik G, Zarbalis KS. Prenatal Neurogenesis in Autism Spectrum Disorders. Front Chem. 2016;4.

6. Thomas MA, Klaper RD: Psychoactive Pharmaceuticals Induce Fish Gene Expression Profiles Associated with Human Idiopathic Autism. Plos One. 2012;7(6).

7. Calisto V, Esteves VI. Psychiatric pharmaceuticals in the environment Chemosphere. 2009:77(10):1257-74

8. Kaushik G, Huber DP, Aho K, Finney B, Bearden S, Zarbalis KS, Thomas MA. Maternal exposure to carbamazepine at environmental concentrations can cross intestinal and placental barriers. Biochem Biophys Res Commun. 2016; 474(2):291-5.

9. Subramanian A, Tamayo P, Mootha VK, Mukherjee S, Ebert BL, Gillette MA, Paulovich A, Pomeroy SL, Golub TR, Lander ES, et al. Gene set enrichment analysis: A knowledge-based approach for interpreting genome-wide expression profiles. Proc Natl Acad Sci U S A. 2005;102(43):15545-50.

10. Kaushik G, Thomas MA, Aho KA. Psychoactive pharmaceuticals as environmental contaminants may disrupt highly inter-connected nodes in an Autism-associated protein-protein interaction network. BMC Bioinformatics. 2015:16.

11. Harden CL. In Utero Valproate Exposure and Autism: Long Suspected, Finally Proven. Epilepsy Curr. 2013;13(6):282-+.

12. Jain P, Cerone MA, LeBlanc AC, Autexier C. Telomerase and neuronal marker status of differentiated NT2 and SK-N-SH human neuronal cells and primary human neurons. J Neurosci Res. 2007:85(1):83-9.

13. Preis PN, Saya H, Nadasdi L, Hochhaus G, Levin V, Sadee W. Neuronal celldifferentiation of human neuro-blastoma cells by retinoic acid plus herbimycin-A. Cancer Res. 1988;48(22):6530-4.

14. Pizzi M, Boroni F, Bianchetti A, Moraitis C, Sarnico I, Benarese M, Goffi F, Valerio A, Spano P. Expression of functional NR1/NR2B-type NMDA receptors in neuronally differentiated SK-N-SH human cell line. Eur J Neurosci. 2002:16(12):2342-50.

15. Kiang TKL, Teng XW, Karagiozov S, Surendradoss J, Chang TKH, Abbott FS. Role of Oxidative Metabolism in the Effect of Valproic Acid on Markers of Cell Viability, Necrosis, and Oxidative Stress in Sandwich-Cultured Rat Hepatocytes. Toxicol Sci. 2010;118(2):501-9.

16. Jones $\sqcup$, Gray M, Yue ST, Haugland RP. Singer VL. Sensitive determination of cell number using the CyQUANT (R) cell proliferation assay. J Immunol Methods. 2001:254(1-2):85-98.

17. Kim D, Pertea G, Trapnell C, Pimentel H, Kelley R, Salzberg SL. TopHat2: accurate alignment of transcriptomes in the presence of insertions, deletions and gene fusions. Genome Biol. 2013;14(4).
18. Team RC. R: A Language and Environment for Statistical Computing. In.i 2012: R Foundation for Statistical Computing.

19. Love MI, Huber W, Anders S. Moderated estimation of fold change and dispersion for RNA-Seq data with DESeq2. 2014. In. bioRxiv.

20. Luo W, Friedman MS, Shedden K, Hankenson KD, Woolf PJ. GAGE: generally applicable gene set enrichment for pathway analysis. BMC Bioinformatics. 2009;10.

21. Briscoe J, Pierani A, Jessell TM, Ericson J. A homeodomain protein code specifies progenitor cell identity and neuronal fate in the ventral neural tube. Cell. 2000;101(4):435-45.

22. Jessell TM. Neuronal specification in the spinal cord: Inductive signals and transcriptional codes. Nat Rev Genet. 2000;1(1):20-9.

23. Toro R, Konyukh M, Delorme R, Leblond C, Chaste P, Fauchereau F, Coleman M, Leboyer M, Gillberg C, Bourgeron T. Key role for gene dosage and synaptic homeostasis in autism spectrum disorders. Trends Genet. 2010; 26(8):363-72.

24. Ghosh A, Michalon A, Lindemann L, Fontoura P, Santarelli L. Drug discovery for autism spectrum disorder: challenges and opportunities. Nat Rev Drug Discov. 2013:12(10):777-90.

25. Benson DL, Colman DR, Huntley GW. Molecules, maps and synapse specificity. Nat Rev Neurosci. 2001;2(12):899-909.

26. Goda Y, Davis GW. Mechanisms of synapse assembly and disassembly. Neuron. 2003;40(2):243-64

27. Airhart MJ, Lee DH, Wilson TD, Miller BE, Miller MN, Skalko RG. Movement disorders and neurochemical changes in zebrafish larvae after bath exposure to fluoxetine (PROZAC). Neurotoxicol Teratol. 2007:29(6):652-64.

28. Hiemke C, Hartter S. Pharmacokinetics of selective serotonin reuptake inhibitors. Pharmacol Ther. 2000:85(1):11-28.

29. Lopez-Figueroa AL, Norton CS, Lopez-Figueroa MO, Armellini-Dodel D, Burke S, Akil H, Lopez JF, Watson SJ. Serotonin 5-HT1A, 5-HT1B, and 5-HT2A receptor mRNA expression in subjects with major depression, bipolar disorder, and schizophrenia. Biol Psychiatry. 2004;55(3):225-33.

30. Margeta MA, Shen K. Molecular mechanisms of synaptic specificity. Mol Cell Neurosci. 2010;43(3):261-7.

31. Bourgeron T. A synaptic trek to autism. Curr Opin Neurobiol. 2009:19(2):231-4.

32. Baudouin SJ, Gaudias J, Gerharz S, Hatstatt L, Zhou K, Punnakkal P, Tanaka KF, Spooren W, Hen R, De Zeeuw Cl, et al. Shared synaptic pathophysiology in syndromic and nonsyndromic rodent models of autism. Science. 2012; 338:128-32.

33. Hough CJ, Irwin RP, Gao XM, Rogawski MA, Chuang DM. Carbamazepine inhibition of N-methyl-D-aspartate-evoked calcium influx in rat cerebellar granule cells. J Pharmacol Exp Ther. 1996;276(1):143-9.

34. Aluru N, Deak KL, Jenny MJ, Hahn ME. Developmental exposure to valproic acid alters the expression of microRNAs involved in neurodevelopment in zebrafish. Neurotoxicol Teratol. 2013;40:46-58.

35. Cox MAA, Cox TF. Multidimensional Scaling. In: Handbook of Data Visualization. Springer Berlin Heidelberg; 2008: 315-347.

36. Lu L, Guo H, Peng Y, Xun G, Liu Y, Xiong Z, Tian D, Liu Y, Li W, Xu X, et al. Common and rare variants of the THBS1 gene associated with the risk of autism. In.: Psychiatric Genetics; 2014.

37. Braunewell KH, Dwary AD, Richter F, Trappe K, Zhao C, Giegling I, et al. Association of VSNL1 with schizophrenia, frontal cortical function, and biological significance for its gene product as a modulator of CAMP levels and neuronal morphology. Transl Psychiatry. 2011;1.

38. Yoshida M, Takayanagi $Y$, Inoue K, Kimura T, Young LJ, Onaka T, Nishimori K. Evidence That Oxytocin Exerts Anxiolytic Effects via Oxytocin Receptor Expressed in Serotonergic Neurons in Mice. J Neurosci. 2009;29(7):2259-71. 